\title{
The Application of Cognitive-Behaviour Therapy in Altering Illness Representations of Systemic Lupus Erythematosus
}

\author{
Donna Goodman \\ James Cook University, Australia \\ Shirley Morrissey \\ Griffith University, Australia \\ Deborah Graham \\ James Cook University, Australia \\ David Bossingham \\ Cairns Base Hospital, Cairns, Australia
}

\begin{abstract}
There is evidence to suggest that illness representations are associated with chronic illness outcomes. Accordingly, several authors have recommended that interventions aimed at improving illness outcomes should include an illness-representations component. While a few researchers have attempted to develop such interventions for chronic illness and chronic pain, no such intervention has been developed for individuals with systemic lupus erythematosus (SLE), a chronic auto-immune illness which results in a variety of negative physical and psychological symptoms. This article reports on a pilot program that investigated the feasibility of a cognitive and behavioural-based intervention for treating SLE which included an illness-representations-change component. The effectiveness of the intervention compared to usual treatment for SLE was evaluated. Also investigated was whether changed illness representations had a beneficial effect on physical health and psychological wellbeing. It was found that the intervention did change participants' treatment control and emotional representations, and that perceived stress was reduced following the intervention. The importance of these findings is discussed, two rival hypotheses for the findings obtained are explored and directions for future research are suggested.
\end{abstract}

Tn studies into the structure of the illness-representations stage of the self-regulatory model (Leventhal, Meyer, \& Nerenz, 1980), it has consistently been found that individuals organise their experiences around five components: (a) identity - the label the person uses to describe the illness and the symptoms associated with it, (b) cause - personal ideas about the cause of the illness, for example, as a result of a viral infection, genetic factors or environmental pollutants, (c) consequences - expected short- and long-term outcomes of the illness, (d) timeline - how long the patient

Address for correspondence: Dr Donna Goodman, PO Box 6343, Cairns QLD 4870, Australia.

E-mail: Donna.Goodman@jcu.edu.au 
believes the illness will last, and (e) cure or control — the person's ideas about what they or healthcare providers can do to influence the course of the illness (Scharloo \& Kaptein, 1997).

More recently, the original conceptualisation of the illness-representations stage of the model was updated to include several changes (Moss-Morris et al., 2002). Specifically, the control/cure component was divided into two separate scales, with one measuring beliefs about personal control, and the other measuring beliefs about treatment control. Similarly, a second timeline scale was added, timeline cyclical, which measures beliefs regarding the cyclical nature of an illness. Finally, two new domains were added: illness coherence, which attempts to assess the extent to which the illness representations developed by the individual provide a coherent framework for understanding the illness, and emotional representations, which are formed in parallel with cognitive illness representations and which are distinct from the individual's general mood in that they are emotional responses generated specifically by the illness rather than by other factors (Moss-Morris et al., 2002).

These components have been used in several studies to establish the structure and specific content of the illness representations of a variety of chronic illnesses, including diabetes (Hampson, Glasgow, \& Toobert, 1990); rheumatoid arthritis, chronic fatigue syndrome and pain (Weinman, Petrie, Moss-Morris, \& Horne, 1996); myocardial infarction (Petrie, Weinman, Sharpe, \& Buckley, 1996); chronic obstructive pulmonary disease and psoriasis (Scharloo et al., 1998); multiple sclerosis (Schiaffino, Shawaryn, \& Blum, 1998); Addison's disease, (Heijmans, 1999); and asthma and HIV (Moss-Morris et al., 2002).

The relationships between illness representations and illness outcomes have also been investigated in many of these studies. The results of these studies have confirmed previous findings that individuals regulate their health-related behaviours according to the beliefs they hold in regard to the specific components of their illness representations (Scharloo \& Kaptein, 1997). As a result of these findings, interventions that take into account the illness-representations stage of the self-regulatory model have been developed as a way of improving illness outcomes (Cooper, Lloyd, Weinman, \& Jackson, 1999; Edwards, Suresh, Lynch, Clarkson, \& Stanley, 2001; Glasgow, Hampson, Stryker, \& Ruggiero, 1997; Heijmans, 1999; Pimm, Byron, Curson, \& Weinman, 1994; Steed, Newman, \& Hardman, 1999). However, very few studies that report on the development of such interventions have been published. The results of those studies that have been published demonstrate that interventions incorporating appropriate restructuring of illness cognitions of several different chronic conditions can enhance the ability of those interventions to improve illness outcomes (Deale, Chalder, \& Wessely, 1998; Jensen, Turner, \& Romano, 2001; Lipchik, Milles, \& Covington, 1993; Petrie, Cameron, Ellis, Buick, \& Weinman, 2000; Pimm et al., 1994; Rainville, Ahern, \& Phalen, 1993).

These findings also highlight the fact that relationships exist between beliefs about illnesses and outcome measures of mood and behaviour in chronic illnesses. This finding is very useful, as it suggests that the predictions of the self-regulatory model are consistent with the theories that underlie cognitive-behavioural interventions for mood disorders which also posit the existence of relationships between beliefs, emotions and behaviour (Clark \& Fairbairn, 1996). For this reason, Petrie and Weinman (1997) suggest that researchers developing interventions aimed at changing illness representations should base such interventions on established cognitive-behavioural techniques. 
Systemic lupus erythematosus (SLE) is a chronic illness in which the normal functioning of the immune system is disrupted. Individuals with SLE report a range of physical symptoms. However, psychological and psychiatric symptoms are also reported. These include psychosis, depression, anxiety and adjustment disorder (Shortall, Isenberg, \& Newman, 1995; Stoll et al., 2001). Other emotional responses experienced include stress and anger (Adams, Dammers, Saia, Brantley, \& Gaydos, 1994); emotional distress; feelings of uncertainty, helplessness, lack of control and loss of confidence (Failla, Kuper, Nick \& Lee, 1996); and fear of death (Liang et al., 1984).

While a literature exists regarding the symptomatology of SLE, there has been very little research into the illness representations of individuals with SLE, and the development of an intervention that attempts to address illness representations of SLE and thereby improve SLE illness outcomes has not been reported on in any studies. Thus, the current study was designed as a pilot project to provide some preliminary information regarding the development of such an intervention. Two research questions were posed: (a) is it possible to change the illness representations of individuals with SLE? and (b) are changes associated with improved illness outcomes?

\section{Method}

\section{Design}

Since this was not a true randomly allocated treatment/control group design, a twogroup comparison design consisting of an intervention and a contrast group was used (Wilkinson, 1999). This repeated-measures within- and between-subjects design allowed for measurement of the change that occurred as a result of the intervention, while controlling for naturally occurring change over time in the variables of interest as well as participants' baseline scores on all measures. The interventiongroup results were compared to the results of a contrast group of individuals with SLE who received only treatment as usual.

\section{Participants}

Thirty-three participants self-selected into either the intervention group $(N=11)$ or the contrast group $(N=22)$. The only significant difference between the groups on medical indices at the start of the study was current illness activity as measured by the British Isles Lupus Assessment Group Index (BILAG; Hay, 1995). In addition, the intervention-group participants had significantly higher scores on measures of anxiety, depression and stress. For the intervention group, mean anxiety scores were in the clinical range. All 11 participants who commenced the intervention completed it; therefore, process data are reported for 11 participants. The 22 participants who did not take part in the intervention continued with their usual treatment regime. All 33 participants completed baseline and post-intervention measures, but 8 participants did not return 12-month follow-up data. Therefore, group comparisons are presented for 15 contrast-group participants and 10 intervention-group participants.

\section{Measures}

Five outcome measures were employed for this study. Physical health status was measured using the SF-36 Physical Health Component Summary (SF-36; Shapiro, 1996) and the Fatigue Intensity Scale (FIS; Maisiak \& Overman, 1998). The SF-36 
is recommended for the measurement of individuals' perceptions of SLE, as these frequently differ from the clinician's view and therefore need to be assessed independently of clinical measures (Isenberg \& Ramsey-Goldman, 1999). The questionnaire contains 36 items which form eight multi-item domains, including physical functioning (10 items), role - physical ( 4 items), bodily pain ( 2 items), general health (5 items), vitality ( 4 items), social function ( 2 items), role - emotional (3 items) and mental health ( 5 items). Scores for each domain range from 0 to 100 , with higher scores indicating better health. The second measure of physical health status, the FIS, contains eight items that are scored on a 4-point Likert scale. Responses include strongly agree (4), slightly agree (3), slightly disagree (2) and strongly disagree (1). Raw scores are calculated and converted into an interval measure, with higher scores indicating greater fatigue.

Psychological distress was measured using the Hospital Anxiety and Depression Scale (HADS; Zigmond \& Snaith, 1983) and the Perceived Stress Scale (PSS; Cohen, Kamarck, \& Mermelstein, 1983). The HADS is a 14-item self-report scale that provides a brief state measure of anxiety (seven items) and depression (seven items). Items are scored from 0 to 3 or 3 to 0 depending on the direction of the item wording (Bowling, 1994). Total scores range from 0 to 21 for each scale. Higher scores indicate more-severe symptomatology. The PSS also consists of 14 items that are scored on a 5-point scale, where choices include never (0), almost never (1), sometimes (2), fairly often (3) and very often (4). Higher scores indicate more-negative perceptions of stress.

Illness representations were measured using the Illness Perceptions Questionnaire Revised (IPQ-R; Moss-Morris et al., 2002). The IPQ-R was used to derive the components of the participants' SLE illness representations, and was made specific to SLE by replacing the words 'your illness' wherever they occurred with 'your SLE'; by adding an additional seven symptoms commonly experienced by people with SLE to the identity scale; and by adding two additional items, hormones and drugs, to the causes scale.

In addition, three process measures were used to monitor changes in participants' psychological distress and cognitive distortions during the intervention. These were the HADS (as described above); the Centre for Epidemiological Studies - Depression Scale (CES-D; Radloff, 1977), a 20-item self-report measure on which respondents are asked to rate the frequency of the occurrence of 20 symptoms in the past week using a 4-point scale ranging from 0 (rarely or none of the time) to 3 (most or all of the time); and the Cognitive Distortion Scale (CDS; Briere, 2000), a brief measure of five types of dysfunctional cognitions: self-criticism, helplessness, hopelessness, self-blame and preoccupation with danger. Each CDS scale contains eight items, giving 40 items in total, which are rated according to their frequency of occurrence over the past month using a 5-point scale ranging from 1 (never) to 5 (very often).

\section{Procedure}

Baseline measures were completed at two time points (July 2000 and November 2000). Four treatment groups were established, each containing between 2 and 4 participants. Each group had two facilitators (registered psychologists), and the intervention consisted of two hour-long sessions separated by a 20-minute rest break. The three process measures were administered at the beginning of each session. The HADS and the CES-D were administered weekly, and the CDS was 
TABLE 1

Elements of the Intervention

\begin{tabular}{|c|c|}
\hline Element & Content \\
\hline Cognitive & $\begin{array}{l}\text { - Socialisation to the cognitive model } \\
\text { - Understanding the role of depression, anxiety and stress } \\
\text { in chronic illness } \\
\text { - Identifying, recording and challenging negative automatic } \\
\text { thoughts, thinking mistakes, assumptions and core beliefs } \\
\text { - Cognitive restructuring } \\
\text { - Symptom management techniques such as relaxation } \\
\text { exercises, distraction, guided imagery and mindfulness } \\
\text { meditation }\end{array}$ \\
\hline Behavioural & $\begin{array}{l}\text { - Behavioural experiments } \\
\text { - Controlled breathing } \\
\text { - Progressive muscle relaxation } \\
\text { - Action plans } \\
\text { - Goal-setting } \\
\text { - Problem-solving }\end{array}$ \\
\hline Illness Representations & $\begin{array}{l}\text { Psychoeducation regarding illness identity, } \\
\text { consequences, personal consequences, emotional } \\
\text { representations and timeline }\end{array}$ \\
\hline
\end{tabular}

administered at weeks 1, 4 and 7. Participants completed the post-intervention questionnaires in March 2001 and July 2001, and a 12-month follow-up in March 2002. In addition to these standard questionnaires, all three process measures (HADS, CES-D and CDS) were administered again at 2-weeks', 4-months' and 12 months' post-intervention.

The intervention was loosely based on the Chronic Illness Self-Management Course developed by Lorig and others (Lorig et al., 2000), and was tailored so that it related specifically to participants with SLE. Two manuals were developed for the treatment group - one leaders' manual and one client workbook. Elements of the intervention are detailed in Table 1.

\section{Results}

Participants in the treatment group were more likely to be single and to have a higher level of education, and less likely to be working part-time, than participants in the contrast group. Mean scores (with standard error bars) for the three process measures for the 11 intervention-group participants are provided in Figures 1 to 3. It is apparent from Figure 1 that anxiety scores were higher than depression scores at all times during the intervention, but that the changes in these scores followed a fairly parallel pattern over time. Also of note from Figure 2 is the appearance that CES-D scores followed a fairly parallel pattern to the HADS scores over time. However, Spearman's rho correlations between these two measures at Week 1 were not significant, $r_{\mathrm{s}}=.43, p=.21$. Correlations between the CES-D and HADS depression scale approached significance, $r_{\mathrm{s}}=.56, p=.09$. Accordingly, it would 


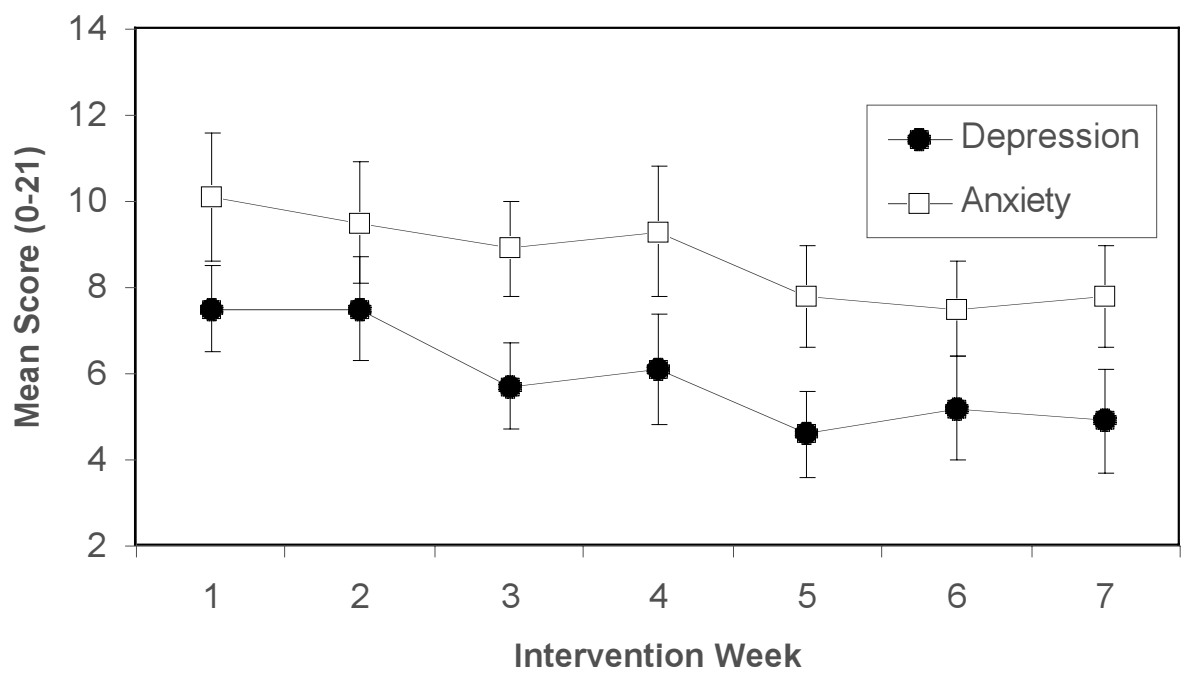

FIGURE 1

Changes in mean Hospital Anxiety and Depression Scale anxiety and depression scores $( \pm \mathrm{SE})$ during the intervention.

Note: $n=11$

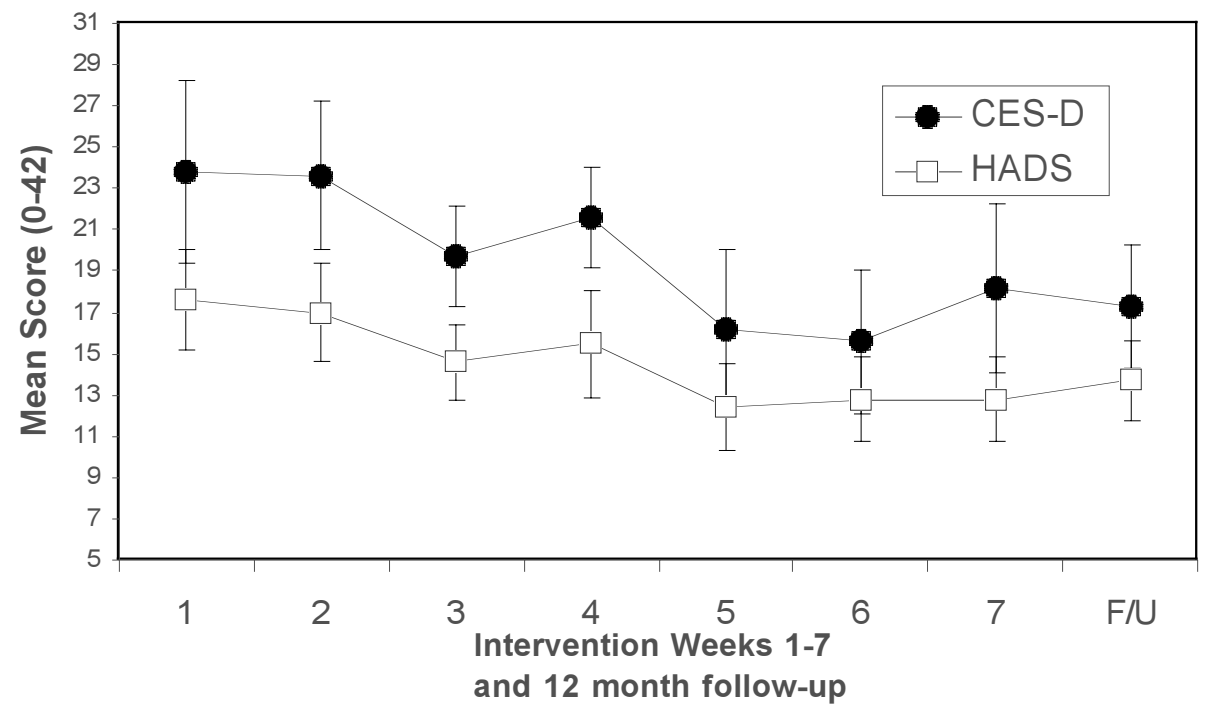

FIGURE 2

Changes in mean Centre for Epidemiological Studies - Depression Scale (range 0-60) and total Hospital Anxiety and Depression Scale (range 0-42) scores ( \pm SE) during the intervention and at 12-month follow-up.

Note: $n=10$

Changes on the Hospital Anxiety and Depression Scale are significant. 


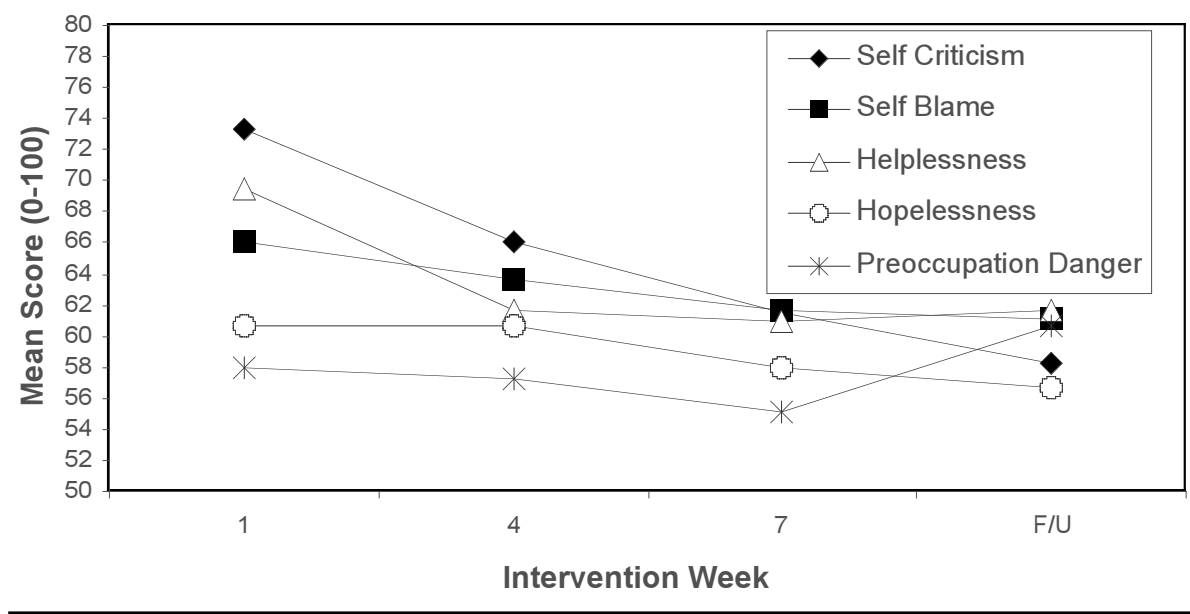

\section{FIGURE 3}

Changes in mean Cognitive Distortion Scale scores at weeks 1, 4 and 7 during the intervention, and at 12-month follow-up.

Note: $n=10$

Changes in self-criticism and helplessness are significant.

appear that these two measures were assessing different aspects of participants' psychological distress. Nonetheless, these data suggest there was a reduction in scores on all process measures during the period of the intervention.

A series of Friedman ANOVAs by ranks was used to determine if changes in process measures were statistically significant. The ranks for HADS anxiety, total HADS scale and CES-D depression all decreased from Week 1 to Week 7, and again at 12-month follow-up, indicating an improvement in psychological distress. However, only the change in the total HADS scale was significant, $\chi_{\mathrm{F}}^{2}=3.2$, $p<.01$. The ranks for HADS depression decreased from Week 1 to Week 7 , but then rose again slightly at follow-up. The ranks for the CDS scales all decreased from Week 1 to Week 7, indicating a decrease in these maladaptive thinking patterns; however, only the changes in self-criticism, $\chi_{\mathrm{F}}^{2}=15.3, p<.002$, and helplessness, $\chi_{\mathrm{F}}^{2}=10.0, p<.01$, were significant. The ranks all rose again slightly at the 12 -month follow-up except for the preoccupation with danger scale, which rose to higher than pre-intervention levels.

The first aim of this study was to determine if the participants' illness representations changed following the intervention. In order to control for naturally occurring changes in illness representations, two sets of baseline and post-intervention measures were obtained for each individual. The mean of the two baseline measures was compared with the mean of the two post-intervention measures. In order to determine if any changes that occurred following the intervention were maintained over time, a set of follow-up measures was obtained at 12-months' post-intervention.

A series of mixed within- and between-subjects MANOVAs was conducted to compare scores on each of the eight illness-representations subscales for the effects of time, group and any interaction between the two. The first variable, time, consisted of three levels: prior to the intervention (baseline), following the intervention 
TABLE 2

Descriptive Statistics for IPQ-R Scales With MANOVA Test Results for Baseline, Post-Intervention and Follow-Up

\begin{tabular}{|c|c|c|c|}
\hline \multirow[t]{2}{*}{$\begin{array}{l}\text { Illness-Representations Subscale } \\
\text { and Time Period }\end{array}$} & \multicolumn{3}{|c|}{$\begin{array}{l}\text { Combined Intervention and Contrast } \\
\text { Groups }(n=25)\end{array}$} \\
\hline & $M$ & $S D$ & $F(d f) p\left(\chi^{2}\right)$ \\
\hline \multicolumn{4}{|l|}{ Identity (Range 0-20) } \\
\hline Baseline & 8.10 & 3.9 & $F(2,22)=4.62$ \\
\hline Post-intervention & 6.58 & 4.6 & $p<.02$ \\
\hline Follow-up & 6.56 & 4.7 & $\left(\eta^{2}=.30\right)$ \\
\hline \multicolumn{4}{|l|}{ Timeline (Acute/Chronic) } \\
\hline Baseline & 3.72 & 0.88 & $F(2,22)=1.61$ \\
\hline Post-intervention & 3.90 & 0.69 & $p<.22$ \\
\hline Follow-up & 3.72 & 0.94 & $\left(\eta^{2}=.13\right)$ \\
\hline \multicolumn{4}{|l|}{ Consequences } \\
\hline Baseline & 2.84 & 0.97 & $F(2,22)=0.33$ \\
\hline Post-intervention & 2.88 & 0.90 & $p<.72$ \\
\hline Follow-up & 2.91 & 0.98 & $\left(\eta^{2}=.03\right)$ \\
\hline \multicolumn{4}{|l|}{ Personal Control } \\
\hline Baseline & 3.66 & 0.61 & $F(2,22)=2.31$ \\
\hline Post-intervention & 3.75 & 0.74 & $p<.12$ \\
\hline Follow-up & 3.83 & 0.62 & $\left(\eta^{2}=.17\right)$ \\
\hline \multicolumn{4}{|l|}{ Treatment Control } \\
\hline Baseline & 3.35 & 0.40 & $F(2,22)=3.68$ \\
\hline Post-intervention & 3.46 & 0.59 & $p<.05$ \\
\hline Follow-up & 3.68 & 0.63 & $\left(\eta^{2}=.25\right)$ \\
\hline \multicolumn{4}{|l|}{ Illness Coherence } \\
\hline Baseline & 3.07 & 0.40 & $F(2,22)=3.14$ \\
\hline Post-intervention & 3.60 & 0.97 & $p<.06$ \\
\hline Follow-up & 3.59 & 0.99 & $\left(\eta^{2}=.22\right)$ \\
\hline \multicolumn{4}{|l|}{ Timeline Cyclical } \\
\hline Baseline & 3.32 & 0.73 & $F(2,22)=0.004$ \\
\hline Post-intervention & 3.31 & 0.94 & $p<.97$ \\
\hline Follow-up & 3.32 & 0.85 & $\left(\eta^{2}=.00\right)$ \\
\hline \multicolumn{4}{|l|}{ Emotional Representations } \\
\hline Baseline & 2.36 & 0.70 & $F(2,22)=3.70$ \\
\hline Post-intervention & 2.18 & 0.57 & $p<.05$ \\
\hline Follow-up & 2.23 & 0.77 & $\left(\eta^{2}=.25\right)$ \\
\hline
\end{tabular}

Note: Scales range from 1 to 5 unless otherwise stated.

(post-intervention) and 12 months later (follow-up). The second variable, group, consisted of two levels: the intervention group and the contrast group. Since no interaction effect for time and group emerged, the means and standard deviations for each illness-representations subscale for the combined sample are presented in Table 2 along with the MANOVA multivariate test results for the effect of time. 
Significant main effects were found for time on the following IPQ-R scales: identity, treatment control and emotional representations. The large effect sizes obtained for each of these analyses indicate that the magnitude of the differences over time was quite substantial. There were also significant main effects for group on the identity, timeline, consequences and emotional representations IPQ-R scales, indicating differences between the intervention group and the contrast group. Again, the large effect sizes obtained for each of these analyses indicate that the magnitude of the differences between the groups was substantial. As stated, there were no significant time by group interaction effects.

In answer to the first research question, it is clear that changes did occur over time on some illness-representations subscales. Large individual differences were evident in these changes. However, these changes were not different for the two groups, which means the intervention failed to demonstrate any significant treatment effects on the illness representations of participants. The exception to this was the illness-coherence subscale for the treatment group, which increased significantly from pre- to post-intervention. No pattern could be detected in the individual change scores, and grouping particular subscales together did not add to the explanatory value of the results.

The second aim of this study was to determine if the intervention produced a significant treatment effect on the outcome measures. In order to examine this question, a series of mixed within- and between-subjects MANOVAs was conducted. Scores on each of the outcome measures were analysed for main effects due to time and group, and for any interaction between the two. As before, the first variable, time, consisted of three levels: prior to the intervention (baseline), following the intervention (post-intervention) and 12 months later (follow-up). The second variable, group, consisted of two levels: intervention group and contrast group. Since no interaction effect for time and group emerged, the means and standard deviations for each outcome measure for the combined sample are presented in Table 3, along with the MANOVA multivariate test results for the effect of time.

Significant main effects were found for time for the PSS, Wilks' Lambda $=0.69$, $F(2,22)=5.041, p<.02$, multivariate eta-squared $=.31$, indicating there was a change in perceived stress across the three time periods. The large effect size obtained for this analysis indicates that the changes were quite substantial. The main effect of time for the total HADS scale was approaching significance, Wilks' Lambda $=0.77, F(2,22)=3.340, p<.054$, multivariate eta-squared $=.23$.

Similarly, significant main effects were found for group for the total HADS scale, $F(1,23)=12.58, p<.002$, multivariate eta-squared $=.35$; the PSS, $F(1,23)$ $=8.67, p<.007$, multivariate eta-squared $=.27$; and the FIS, $F(1,23)=7.43$, $p<.02$, multivariate eta-squared $=.24$, indicating differences between the intervention group and the contrast group on these measures. Again, the large effect sizes indicate that the magnitude of the differences between the groups was substantial. However, there were no significant interaction effects between time and group on any of the outcome measures.

As correlations have been used to demonstrate treatment effects in previous studies that have investigated illness representations, two correlation matrices were developed. Displayed in the first matrix (Table 4) are the intercorrelations between changes in baseline and post-intervention scores on outcome measures. As shown in Table 4, reductions in fatigue were significantly correlated with improved physical health status and reductions in psychological distress. Examined in the second 
TABLE 3

Descriptive Statistics for Outcome Measures With MANOVA Test Results for Baseline, Post-Intervention and Follow-Up

\begin{tabular}{|c|c|c|c|}
\hline \multirow[t]{2}{*}{$\begin{array}{l}\text { Illness-Representations Subscale } \\
\text { and Time Period }\end{array}$} & \multicolumn{3}{|c|}{$\begin{array}{l}\text { Combined Intervention and Contrast } \\
\text { Groups }(n=25)\end{array}$} \\
\hline & $M$ & $S D$ & $F(d f) p\left(\chi^{2}\right)$ \\
\hline \multicolumn{4}{|l|}{ SF-36 Physical Health Status (0-100) } \\
\hline Baseline & 40.07 & 13.02 & $F(2,22)=0.08$ \\
\hline Post-intervention & 39.88 & 13.13 & $p<.92$ \\
\hline Follow-up & 39.76 & 12.78 & $\left(\eta^{2}=.007\right)$ \\
\hline \multicolumn{4}{|c|}{$\begin{array}{l}\text { Total Hospital Anxiety and Depression } \\
\text { Scale (Range 0-42) }\end{array}$} \\
\hline Baseline & 10.44 & 6.32 & $F(2,22)=3.34$ \\
\hline Post-intervention & 9.28 & 5.87 & $p<.054$ \\
\hline Follow-up & 8.52 & 7.08 & $\left(\eta^{2}=.23\right)$ \\
\hline \multicolumn{4}{|l|}{ Fatigue Intensity Measure (0-100) } \\
\hline Baseline & 44.94 & 19.12 & $F(2,22)=0.91$ \\
\hline Post-intervention & 41.87 & 17.97 & $p<.41$ \\
\hline Follow-up & 42.68 & 18.75 & $\left(\eta^{2}=.07\right)$ \\
\hline \multicolumn{4}{|l|}{ Perceived Stress Scale (0-56) } \\
\hline Baseline & 21.94 & 6.31 & $F(2,22)=5.04$ \\
\hline Post-intervention & 19.08 & 7.04 & $p<.02$ \\
\hline Follow-up & 19.96 & 8.44 & $\left(\eta^{2}=.31\right)$ \\
\hline
\end{tabular}

correlation matrix (Table 5) was whether baseline to post-intervention changes in illness-representations subscales were correlated with changes in outcome measures. As shown in Table 5, reductions in the identity and timeline-cyclical subscales were significantly correlated with improvements in physical health status, and reductions in the identity, timeline-cyclical and emotional representations subscales were significantly correlated with reduced fatigue. Increases in the personal control subscale were significantly correlated with reduced fatigue. As would be expected, changes in emotional representations were significantly correlated with changes in psychological distress and perceived distress.

\section{Discussion}

When considering the results of this pilot study, it must be remembered that the sample was very small, the partcipants selected the condition they participated in and the sample was characterised by large individual differences between participants. In addition, individuals who elected to participate in the intervention had significantly higher levels of psychological distress and current illness activity than those who continued treatment as usual. Furthermore, the participants who elected to participate in the intervention had a chronic illness, the course of which was known to fluctuate considerably over time. In a randomised control trial, these factors would cast serious doubts on the validity of the study findings due to the potential for confounding by systematic biases in the sample, and on the ability of the 


\section{TABLE 4}

Intercorrelation Matrix for Baseline to Post-Intervention Change Scores on Outcome Measures (Spearman's rho)

\begin{tabular}{lcccc} 
& $\begin{array}{c}\text { PCS Health } \\
\text { Status }\end{array}$ & $\begin{array}{c}\text { Fatigue Intensity } \\
\text { Measure }\end{array}$ & $\begin{array}{c}\text { Hospital Anxiety } \\
\text { and Depression } \\
\text { Scale }\end{array}$ & $\begin{array}{c}\text { Perceived } \\
\text { Stress } \\
\text { Scale }\end{array}$ \\
\hline SF-36 Health Status & - & - & \\
Fatigue Intensity Measure & $-.58^{* * *}$ & $.35^{*}$ & - & -14 \\
Hospital Anxiety and & .21 & .28 & & - \\
Depression Scale & -.21 & & & \\
Perceived Stress Scale & & & & \\
\hline
\end{tabular}

Note: $n=33$

${ }^{*} p<.05,{ }^{* *} p<.01,{ }^{* *} p<.001$

study findings to be generalised to the larger population of individuals with SLE. However, this study was not a randomised control trial of an intervention, nor did it attempt to establish the efficacy of one intervention over another. Rather, it attempted to establish some baseline data about whether or not the illness representations of these particular individuals could be changed through the use of an intervention designed for that purpose, and whether there would be a corresponding change in outcome measures. The following discussion must be considered within this context.

Following the intervention, significant reductions were evident in self-reports of psychological distress and in some cognitive distortions. There were significant changes over time in the identity, treatment control and emotional representations of participants in the intervention group. Similar findings were obtained in regard to the outcome measures. Significant improvements in participants' perception of stress were found to have occurred over time, and the reduction in psychological distress also approached significance. However, in the absence of a significant

\section{TABLE 5}

Correlation Matrix for Baseline to Post-Intervention Change Scores for IPQ-R Subscales and Outcome Measures for Baseline to Post-Intervention Change Scores (Spearman's rho)

Identity Timeline Conse- Personal Treatment IIIness Cyclical Emotional quence Control Control Coherence Timeline Repre-

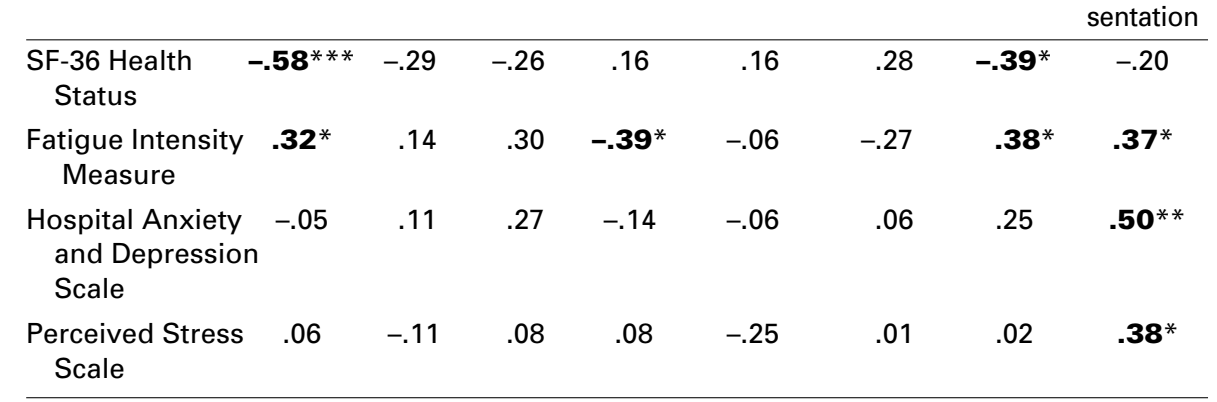

Note: $n=33$

$$
{ }^{*} p<.05,{ }^{*} p<.01,{ }^{* *} p<.001
$$


interaction effect, it cannot be concluded that the observed changes were due to the intervention, as other confounding factors have obviously affected the results.

The finding that the intervention did not produce changes in outcome measures other than perceived stress is at odds with the findings in two previous studies on the development of interventions based on illness representations for chronic illness populations. In the first of these studies, it was found that the intervention produced reductions in pain and functional ability in individuals with arthritis (Pimm et al., 1994), while in the second study, it was found that the intervention group returned to work faster than the control group (Petrie et al., 2000). However, whether the illness representations of participants were measured before and after the intervention was not reported in either of these studies. Instead, correlations between changes in illness-representations subscales and changes in outcome measures were reported. Therefore, no judgment can be made about whether illness representations changed or whether the outcome was due to some other effect. The correlations between illness representations and outcome measures were also examined in the current study, and significant relationships between these variables were found, consistent with the findings of Petrie and colleagues. However, in addition, also measured in the current study were changes in actual illness representations, but in the absence of an interaction effect the authors were unable to demonstrate that these changes were due to the intervention.

The findings of the correlations between fatigue and illness representations, however, are particularly interesting. Fatigue has been found to be one of the most common and debilitating symptoms associated with SLE (Tench, McCurdie, White, \& D'Cruz, 1998), and it has been found that it is unresponsive to drug therapy (Taylor et al., 1998). In addition, in previous medical studies, links between fatigue and measures of illness activity have not been established (Wang, Gladman, \& Urowitz, 1998), but it has been found that individuals with SLE have such diminished aerobic capacity that their ability to perform daily domestic tasks, occupational activities and recreational pursuits is seriously hampered (Keyser et al., 2001). While some researchers have concluded from this evidence that psychosocial factors should be examined to determine if they are a greater predictor of the variance on measures of fatigue than medical indices (Omdal, Mellgren, Koldingsnes, \& Jacobsen, 2001), evidence from the current study suggests that illness representations may also play a role in this regard. Emphasised in the intervention was understanding and managing fatigue and it was apparent that fatigue-intensity scores decreased from baseline to post-intervention, although they returned to baseline levels at 12 -month follow-up.

However, two rival hypotheses could also account for the findings that were obtained. Specifically, it may be that changes in cognitive distortions and/or increased activity levels rather than changes in illness representations per se were responsible for changes in fatigue.

Similarly, it was found that reductions occurred in levels of perception of stress, and also in levels of psychological distress. While significant changes were found in the treatment-control and emotional representations scales targeted by the intervention, significant reductions were again found in the self-criticism and helplessness scales of the CDS. Furthermore, participants recorded increased activity levels on their weekly action plans, although this outcome was not formally measured. There is considerable support in the literature for the argument that both cognitive change and increased physical activity improve depression in nonmedical outpatients 
(Beck, Rush, Shaw, \& Emery, 1979). In addition, it has been demonstrated in studies of individuals with SLE that physical exercise improves psychological wellbeing (Clarke-Jenssen, Fredriksen, Lilleby, \& Mengshoel, 2001; Ramsay et al., 2000; Tench, McCurdie, McCarthy, White, \& D'Cruz, 2001).

The findings from the current study provide at least partial support for the rival hypotheses that reduced cognitive distortions and/or increased activity levels were the effective elements of the intervention. However, these findings need to be examined further as it was not possible in the current study to separate and individually assess the relative contributions of the various elements of the intervention. Nor was it possible to test for the possibility that cognitive distortions or increased activity mediate the relationship between illness representations and outcome measures. What these findings do suggest is that future research into the utility of illness-representations interventions needs to be designed to allow both of these rival hypotheses to be tested and excluded.

Another explanation which could account for the failure of this intervention to produce the expected outcomes in regard to both illness representations and outcome measures may be related to the participants' severity of illness. In previous studies it has been found that psychological interventions are least effective for individuals with more-severe illness, as such individuals find it difficult to concentrate and engage in treatment (Guthrie, 1996). An examination of the physical health-status scores revealed that participants in this study had worse physical health than other SLE cohorts (see for example, Da Costa et al., 2000a). Although the participants reported in their evaluation interview that they had obtained some benefit from the intervention, this benefit may not have been detected by the measures used, or it may not have been large enough to produce a statistically significant result.

In addition to these practical explanations for the results obtained, there are a number of statistical factors that may also have contributed to the results. First, it is possible that the small sample size was responsible for the failure to demonstrate an effect. In other words, Type II errors may have occurred.

Another possibility is that the detection of changes on some scales but not on others was due to the differential strength of the measures to detect changes over time. If this was in fact the case, the summing of two sets of measures to obtain baseline and post-intervention values may also have contributed to the lack of significance in the current study. As SLE is known to exhibit a variable course over time, calculating these mean scores may have obscured differences that were important to the result. Similarly, Type II errors are likely when participants' baseline scores are either at the floor or ceiling of measures on which change is expected to occur. In the current study, a small number of participants recorded either floor or ceiling scores on some illness-representations scales and some outcome measures, particularly on the HADS. Therefore, the level of change recorded would have been deflated to some extent by the limited ability of these scores to change from their original level.

\section{Research Strengths and Limitations}

The use of a small, self-selected sample; self-report measures; and the statistical factors outlined above are the most important limitations of the current study. However, there are a number of other limitations that should be acknowledged. First, the researcher was also one of the leaders for all four intervention groups. 
Separating these roles would have reduced the chance of confounding of results by experimenter effects. While such a course of action was not possible in the current study, a number of steps were taken in an attempt to control for experimenter bias. These included having the researcher present the intervention to all groups so that any effect was the same across groups, having two leaders in each group and having the intervention completely manualised. Future studies should aim to achieve a greater separation of roles as there is no way to estimate the extent of experimenter effects, or to control for these effects with post hoc measures or statistical adjustments. However, it is also acknowledged that the ecological validity of intervention studies is enhanced when such studies are conducted in the same way as occurs in clinical practice (see for example, Seligman, 1995).

In conclusion, the current study is one of only a few studies that have tested whether changes occur in individuals' psychological status following the delivery of an intervention that includes an illness-representations-change component. Although the expected treatment effects did not emerge as a result of the intervention, a number of important findings were made. First, changes were noted in regard to treatment control and emotional representations. Second, some changes in the expected direction were noted in levels of psychological distress and perceived stress. A number of possible explanations were proposed for these findings, a number of questions were raised regarding the analyses conducted and a number of directions for future research were suggested.

\section{References}

Adams, S.G., Dammers, P.M., Saia, T.L., Brantley, P.J., \& Gaydos, G.R. (1994). Stress, depression and anxiety predict average symptom severity in daily symptom fluctuation in systemic lupus erythematosus. Journal of Behavioural Medicine, 17(5), 459-477.

Beck, A.T., Rush, A.J., Shaw, B.F., \& Emery, G. (1979). Cognitive therapy of depression. New York: The Guilford Press.

Bowling, A. (1994). Measuring health: A review of quality of life measurement scales. Philadelphia: Open University press.

Briere, J. (2000a). Cognitive Distortion Scales (CDS) professional manual. Odessa, FL: Psychological Assessment Resources.

Clark, D.M., \& Fairbairn, C.G. (1996). Science and practice of cognitive therapy. Oxford, UK: Oxford University Press.

Clarke-Jenssen, A.C., Fredriksen, P.M., Lilleby, V., \& Mengshoel, A.M. (2005). Effects of supervised aerobic exercise in patients with systemic lupus erythematosus: A pilot study. Arthritis Care EO Research, 53(2), 308-312.

Cohen, S., Kamarck, T., \& Mermelstein, R. (1983). A global measure of perceived stress. Journal of Health and Social Behaviour, 24, 38-396.

Cooper, A., Lloyd, G., Weinman, J., \& Jackson, G. (1999). Why patients do not attend cardiac rehabilitation: Role of intentions and illness beliefs. Heart, 82, 234-236.

Da Costa, D., Dobkin, P.L., Fitzcharles, M-A., Fortin, P.R., Beaulieu, A., Zummer, M., et al. (2000a). Determinants of health status in fibromyalgia: A comparative study with systemic lupus erythematosus. The Journal of Rheumatology, 27, 365-372.

Deale, A., Chalder, T., \& Wessely, S. (1998). Illness beliefs and treatment outcome in chronic fatigue syndrome. Journal of Psychosomatic Research, 45(1), 77-83.

Edwards, R., Suresh, R., Lynch, S., Clarkson, P., \& Stanley, P. (2001). Illness perceptions and mood in chronic fatigue syndrome. Journal of Psychosomatic Research, 50, 65-68.

Failla, S., Kuper, B.C., Nick, T.G., \& Lee, F.A. (1996). Adjustment of women with systemic lupus erythematosus. Applied Nursing Research, 9, 87-93. 
Glasgow, R.E., Hampson, S.E., Stryker, L.A., \& Ruggiero, L. (1997). Personal-model beliefs and social environmental barriers related to diabetes self-management. Diabetes Care, 20(4), $556-561$.

Guthrie, E. (1996). Emotional disorder in chronic illness: Psychotherapeutic interventions. British Journal of Psychiatry, 168, 265-273.

Hampson, S.E., Glasgow, R.E., \& Toobert, D.T. (1990). Personal models of diabetes and their relation to self-care activities. Health Psychology, 9, 632-646.

Hay, E.M. (1995). Systemic lupus erythematosus. Bailliere's Clinical Rheumatology, 9, 437-470.

Heijmans, M. (1999). The role of patients' illness representations in coping and functioning with Addison's disease. British Journal of Health Psychology, 4, 137-149.

Isenberg, D., \& Ramsey-Goldman, R. (1999). Assessing patients with lupus: Towards a drug responder index. Rheumatology, 38, 1045-1049.

Jensen, M.P., Turner, J.A., \& Romano, J.M. (2001). Changes in beliefs, catastrophizing, and coping are associated with improvement in multidisciplinary pain treatment. Journal of Consulting and Clinical Psychology, 69(4), 655-662.

Keyser, R.E., Rus, V., Cade, W.T., Kalappa, N., Flores, R.H., Mikdashi, J.A., et al. (2001, November). Diminished aerobic capacity is a mechanism of fatigue in women with mild systemic lupus erythematosus. Paper presented at the ACR 65th Annual Scientific Meeting, ARHP 36th Annual Scientific Meeting, San Francisco, CA USA.

Leventhal, H., Meyer, D., \& Nerenz, D.R. (1980). The common sense representation of illness danger. In S. Rachman (Ed.), Contributions to medical psychology (Vol. 2, pp. 17-30). New York: Pergamon.

Liang, M.H., Rogers, M., Larson, M., Eaton, H.M., Murawski, B.J., Taylor, J.E., et al. (1984). The psychological impact of systemic lupus erythematosus and rheumatoid arthritis. Arthritis and Rheumatism, 27, 13-19.

Lipchik, G.L., Milles, K., \& Covington, E.C. (1993). The effects of multidisciplinary pain management treatment on locus of control and pain beliefs in chronic non-terminal pain. The Clinical Journal of Pain, 9, 49-57.

Lorig, K., Holman, H., Sobel, D., Laurent, D., Gonzalez, V., \& Minor, M. (2000). Living a healthy life with chronic conditions: Self-management of heart disease, arthritis, diabetes, asthma, bronchitis, emphysema and others. Palo Alto, CA: Bull Publishing Company.

Maisiak, R., \& Overman, B.S. (1998). Development and testing of the fatigue intensity scale (FIS) using rasch analysis. Arthritis and Rheumatism, 41, 9(Suppl.), S290.

Moss-Morris, R., Weinman, J., Petrie, K.J., Horne, R., Cameron, L.D., \& Buick, D. (2002). The revised illness perception questionnaire (IPQ-R). Psychology and Health, 17(1), 1-16.

Omdal, R., Mellgren, S.I., Koldingsnes, W., \& Jacobsen, E.A. (2002). Fatigue in patients with systemic lupus erythematosus: Lack of associations to serum cytokines, antiphospholipid antibodies, or other disease characteristics. Journal of Rheumatology, 29, 482-486.

Petrie, K. J. \& Weinman, J. (1997). Perceptions of health and illness: Current research and applications. Amsterdam: Harwood Academic Publishers.

Petrie, K.J., Cameron, L.D., Ellis, C., Buick, D., \& Weinman, J. (2002). Changing illness perceptions after myocardial infarction: An early intervention randomized controlled trial. Psychosomatic Medicine, 64(4), 580-586.

Petrie, K., Weinman, J., Sharpe, N., \& Buckley, J. (1996). Role of patient's view of their illness in predicting return to work and functioning after myocardial infarction: Longitudinal study. British Medical Journal, 312, 1191-1194.

Pimm, T.J., Byron, M.A., Curson, D., \& Weinman, J. (1994b). Personal illness models and the self management of arthritis. Arthritis and Rheumatism, 37, 9(Suppl.), S358.

Radloff, L. (1977). The CES-D Scale: A self-report depression scale for research in the general population. Applied Psychosocial Measurement, 1, 385-401.

Rainville, J., Ahern, D.K., \& Phalen, L. (1993). Altering beliefs about pain and impairment in a functionally oriented treatment program for chronic low back pain. The Clinical Journal of Pain, 9, 196-201. 
Ramsay, C., Moreland, J., Ho, M., Joyce, S., Walker, S., \& Pullar, T. (2000). An observer-blinded comparison of supervised and unsupervised aerobic exercise regimens in fibromyalgia. British Journal of Rheumatology, 39, 501-505.

Scharloo, M., \& Kaptein, A. (1997). Measurement of illness perceptions in patients with chronic somatic illnesses: A review. In K.J. Petrie \& J.A. Weinman (Eds.), Perceptions of health and illness: Current research and applications (pp. 103-154). Amsterdam, the Netherlands: Harwood Academic Publishers.

Scharloo, M., Kaptein, A.A., Weinman, J., Hazes, J.M., Willems, L.N., Bergman, W., et al. (1998). Illness perceptions, coping and functioning in patients with rheumatoid arthritis, chronic obstructive pulmonary disease and psoriasis. Journal of Psychosomatic Research, 44, 573-585.

Schiaffino, K.M., Shawaryn, M.A., \& Blum, D. (1998). Examining the impact of illness representations on psychological adjustment to chronic illness. Health Psychology, 17, 262-268.

Seligman, M.E.P. (1995). The effectiveness of psychotherapy: The Consumer Reports study. American Psychologist, 50, 965-974.

Shapiro, H.S. (1996). Depression in lupus. Retrieved October 3, 1999, from http://www.hamline.edu:80/lupus/articles/Depression_in_Lupus.html

Shortall, E., Isenberg, D., \& Newman, S.P. (1995). Factors associated with mood and mood disorders in SLE. Lupus, 4, 272-279.

Steed, L., Newman, S.P., \& Hardman, S.M.C. (1999). An examination of the self-regulation model in atrial fibrilation. British Journal of Health Psychology, 4, 337-347.

Stoll, T., Kauer, Y., Buchi, S., Klaghofer, R., Sensky, R., \& Villiger, P.M. (2001). Prediction of depression in systemic lupus erythematosus patients using SF-36 mental health scores. Rheumatology, 40, 695-698.

Taylor, J., Sutcliffe, N., Clunie, G., Hakim, A., FitzClarence, H., \& Isenberg, D. (1998). Lupus patients with fatigue: Is there a link with the fibromyalgia syndrome? British Journal of Rheumatology, 37(Suppl. 1), 67.

Tench, C.M., McCarthy, J., McCurdie, I., White, P.D., \& D'Cruz, D.P. (2003). Fatigue in systemic lupus erythematosus: A randomized controlled trial of exercise. Rheumatology (Oxford), 42, 1050-1054.

Tench, C.M., McCurdie, I., White, P.D., \& D'Cruz, D. (1998). The prevalence and associations of fatigue in outpatients with SLE - The FLOP study. British Journal of Rheumatology, 37(Suppl. 1), 68 .

Wang, B., Gladman, D.D., \& Urowitz, M.B. (1998). Fatigue in lupus is not correlated with disease activity. The Journal of Rheumatology, 25, 892-895.

Weinman, J., Petrie, K., Moss-Morris, R., \& Horne, R. (1996). The illness perception questionnaire: A new measure for assessing the cognitive representation of illness. Psychology and Health, 11, 431-445.

Wilkinson, I. (1999). Statistical methods in psychology journals. American Psychologist, 54, 594-604.

Zigmond, A.S., \& Snaith, R.P. (1983). The Hospital Anxiety and Depression Scale. Acta Psychiatrica Scandinavica, 67, 361-370. 